\section{$\underset{\substack{\text { hommes } \\ \text { \& migrations }}}{ }$}

\section{Hommes \& migrations}

Revue française de référence sur les dynamiques

migratoires

Les mots de l'exil dans l'Europe du XIXe siècle

\title{
Immigrations postcoloniales, minorités et politique en France et en Angleterre
}

Perspectives comparatives

\section{Adèle Momméja}

\section{(2) OpenEdition}

Journals

Édition électronique

URL : http://journals.openedition.org/hommesmigrations/4402

DOI : ERREUR PDO dans /localdata/www-bin/Core/Core/Db/Db.class.php L.34 : SQLSTATE[HY000]

[2006] MySQL server has gone away

ISSN : 2262-3353

Éditeur

Musée national de l'histoire de l'immigration

Édition imprimée

Date de publication : 1 avril 2018

Pagination : 127-135

ISBN : 978-2-919040-41-4

ISSN : $1142-852 X$

\section{Référence électronique}

Adèle Momméja, «Immigrations postcoloniales, minorités et politique en France et en Angleterre », Hommes \& migrations [En ligne], 1321 | 2018, mis en ligne le 01 avril 2018, consulté le 08 janvier 2021. URL : http://journals.openedition.org/hommesmigrations/4402 ; DOI : https://doi.org/10.4000/ hommesmigrations.4402 


\title{
IMMIGRATIONS POSTCOLONIALES MINORITÉS ET POLITIQUE EN FRANCE ET EN ANGLETERRE
}

\section{PERSPECTIVES COMPARATIVES}

\begin{abstract}
Par ADÈLE MOMMÉJA, postdoctorante en sociologie, Institut d'histoire du temps présent, université Paris 8.
\end{abstract}

D epuis la fin des années 1970, de nombreuses études ont comparé les politiques d'immigration et d'intégration des immigrés et de leurs descendants en France et en Angleterre. La récurrence de ces analyses comparatives n'est pas surprenante tant ces deux nations européennes s'offrent presque naturellement au regard comparatif. Les années d'après-guerre marquent un afflux massif de populations issues des anciens pays colonisés qui transforment la structure des flux migratoires auparavant composés de populations d'origine européenne. Les deux anciens empires coloniaux mettent aussi en œuvre des politiques de régulation de ces flux et restreignent progressivement les possibilités d'accès à la nationalité. Les modèles d'intégration mis en œuvre en France et en Angleterre reposent néanmoins sur des conceptions différentes des rapports entre l'État et les populations immigrées. Les analyses opposent classiquement le multiculturalisme anglais - où l'intégration est conçue comme l'organisation étatique d'une cohabitation entre la population majoritaire et les populations immigrées - et le modèle républicain qui prescrit l'absence de prise en compte des spécificités ethniques des citoyens par les institutions. Cette dichotomie est-elle réellement opératoire pour décrire la gestion politique des migrations et les parcours des immigrés et de leurs descendants? Comment les chercheurs français et britanniques ont-ils mobilisé, interrogé, critiqué et relativiséces deux modèles ? Peut-on identifier des spécificités nationales dans la gestion étatique de l'immigration, les modes de protestation des immigrés et les dispositifs étatiques de lutte contre les discriminations? Ce texte propose des hypothèses pour répondre à ces questions à partir d'une synthèse comparative des travaux de recherche sur l'intégration sociale et politique des immigrés dans ces deux anciens empires coloniaux?.

\section{L'immigration comme problème public. Genèse des politiques migratoires}

Dès la fin des années 1970, des études françaises et britanniques se sont attachées à comparer les modèles français et britannique d'intégration des 
immigrés ${ }^{2}$. La présence de populations que l'on appelle "travailleurs immigrés" en France et " guest workers » en Angleterre fut d'abord pensée comme provisoire. Jusqu'aux années 1980, l'idée d'un retour prochain au pays d'origine organise autant la vie familiale des immigrés que la gestion de l'immigration par les agents de l'État. La progressive reconnaissance du caractère permanent de l'immigration postcoloniale entraîne la fin de ce "mythe du retour ${ }^{3}$ " et transforme les perceptions privées et publiques de la présence immigrée. Des études socio-historiques ont également exploré le rôle des administrations dans la genèse d'un problème public de l'immigration. Le rôle des hauts fonctionnaires ${ }^{4}$, des préfectures ${ }^{5}$ et des municipalités ${ }^{6}$ dans "l'invention de l'immigra$t_{i o n}{ }^{7}$ » a été mis en évidence.

Les gouvernements français et anglais adoptent, par ailleurs, une politique à double volet qui consiste, d'une part, à restreindre les flux migratoires en exerçant des contrôles accrus aux frontières et, d'autre part, à organiser l'intégration des immigrés légalement installés sur le territoire. La période 1978-1980 marque un moment où l'action publique est fondée sur le préjugé de « l'inassimilabilité » dans la nation française du fait de l'origine culturelle ou religieuse de l'immigration nordafricaine $^{8}$. Le gouvernement de François Mitterrand entame une campagne de légalisation des immigrés illégaux vite suivie par un durcissement de la politique migratoire à l'égard des immigrés clandestins. Dans le même temps, les enfants d'immigrés sont présentés comme la " génération clef » et sont constitués comme la population cible de nouvelles politiques d'intégration ${ }^{9}$. Ces politiques d'orientations contradictoires aboutissent finalement à un "compromis bipartisan " qui combine contrôle des entrées, garantie des droits des résidents en règle, coopération européenne et demeure en place jusque dans les années $1990^{10}$. La période marque également l'apparition de partis xénophobes qui font campagne sur la thématique de l'envahissement des immigrés. En France, le Front national réalise ses premières percées électorales au début des années 1980 et s'installe dans le paysage politique. Ce parti parvient aussi à imposer ses thématiques qui sont reprises par les médias et les partis de toute obédience ${ }^{11}$. Certaines mairies communistes, traditionnellement associées à la défense des populations immigrées, mettent en place des pratiques discriminatoires vis-à-vis de leurs habitants immigrés accusés de faire fuir leur électorat traditionnel issu de la classe ouvrière blanche ${ }^{12}$. Cette légitimation des thématiques de l'extrême droite s'accompagne d'une dénonciation du racisme populaire qui vise notamment à justifier les politiques d'immigration restrictives mises en place depuis le milieu des années $1970^{13}$. En Angleterre, la création, en 1967, du National Front, un parti qui prône la pureté raciale et une idéologie anti-immigration, marque l'institutionnalisation de cette idéologie xénophobe. Mais si l'impact de ce parti sur la scène politique est grandissant à la fin des années 1970,

\footnotetext{
2. Gary P. Freeman, Immigrant Labor and Racial Conflict in Industrial Societies: The French and British Experience, 1945-1975, Princeton, Princeton University Press, 1979 ; Didier Lapeyronnie, L'individu et les minorités: la France et la Grande-Bretagne face à leurs immigrés, Paris, Presses universitaires de France, 1993; Adrian Favell, Philosophies of integration: Immigration and the idea of citizenship in France and Britain, Basingstoke, Palgrave-Macmillan, 1998 ; Erik Bleich, Race Politics in Britain and France: Ideas and Policymaking since the 1960 , Cambridge, Cambridge University Press, 2003. 3. Abdelmalek Sayad, La double absence. Des illusions de l'émigré aux souffrances de l'immigré, Paris, Seuil, 1999. 4. Sylvain Laurens, Une politisation feutrée: les hauts fonctionnaires et l'immigration en France, 1962-1981, Paris, Belin, 2009. 5. Françoise de Barros, "L'État au prisme des municipalités : une comparaison historique des catégorisations des étrangers en France (1919-1984) ", thèse de doctorat, Paris, Université Panthéon-Sorbonne, 2004. 6. Alexis Spire, Étrangers à la carte. L'administration de l'immigration en France, Paris, Grasset \& Fasquelle, 2005. 7. "L'invention de l'immigration ", numéro spécial, Agone, $n^{\circ}$ 40, 2008. 8. Patrick Weil, Liberté, égalité, discriminations. L' identité nationale " au regard de l'histoire, Paris, Folio, 2009. 9. Adèle Momméja, "Les enfants d'immigrés au temps du droit à la différence. Socio-histoire d'une politique compassionnelle ", thèse de doctorat de sociologie, Nanterre, Université Paris-Nanterre, 2016. 10. Philippe Rygiel, " Les politiques d'immigration en France des années 1970 aux années 1990 ", in Cahiers de l'institut CGT d'histoire sociale, 2013, p. 22-26. 11. Simone Bonnafous, L'immigration prise aux mots les immigrés dans la presse au tournant des années 80, Paris, Kimé, 1991. 12. Olivier Masclet, " Une municipalité communiste face à l'immigration algérienne et marocaine ", in Genèses, n 45,2001, pp. 150-163. 13. Sylvain Laurens, "Le racisme, attribut du populaire ? ", in Plein droit, $n^{\circ} 69,2012$, pp. 9-12
} 
il ne parvient pas à s'installer durablement dans le paysage politique. Cette influence limitée s'explique notamment par l'arrivée de Margaret Thatcher au pouvoir et par sa reprise des thèmes anti-immigration et sécuritaire ${ }^{14}$.

\section{Multiculturalisme et intégration républicaine : des modèles opératoires?}

Les années d'après-guerre marquent également l'émergence de politiques d'intégration des immigrés dont la présence permanente est dorénavant reconnue par les pouvoirs publics. En France comme en Angleterre, les conditions d'accès à la citoyenneté furent longtemps relativement souples grâce à l'application du principe juridique du jus soli (droit du sol), introduit en France par la loi de 1889, qui accorde la nationalité aux enfants d'immigrés nés sur leur territoire du pays d'accueil. Le droit du sol fait néanmoins l'objet de remises en cause successives dans ces deux pays à partir des années 1970 et 1980. En France, les années 1980 marquent une résurgence des conceptions ethniques de la nationalité, liée notamment à l'installation du Front national dans le paysage politique français ${ }^{15}$. Le projet de réforme du code de la nationalité, présenté par le gouvernement de Jacques Chirac en octobre 1986, limite la possibilité d'accès des enfants d'immigrés en instaurant l'obligation pour un enfant né en France de faire une demande expresse de nationalité française entre 16 et 19 ans accompagnée d'une prestation solennelle de serment en public ${ }^{16}$. En 1993, une réforme instaure l'obligation, pour les étrangers nés en France et y résidant, d'effectuer une démarche administrative, dite " manifestation de volonté », entre 16 et 21 ans pour devenir français.

En Angleterre, un durcissement législatif entraîne également des remises en cause successives du droit du sol. En 1971, le gouvernement conservateur d'Edward Hearth propose une réforme du droit de l'immigration qui lie l'accession au statut de citoyen à des conditions de maîtrise de la langue anglaise, de "bonne réputation " et de projet d'installation au Royaume-Uni. Dix ans plus tard, le gouvernement Thatcher propose une réforme de la nationalité qui fait évoluer l'ancien droit du sol par celui de la descendance. Les enfants nés au Royaume-Uni de parents étrangers n'obtiennent plus la nationalité automatiquement mais après dix ans de résidence ininterrompue sur le territoire $^{17}$. Les dispositions actuelles du droit de la nationalité britannique héritent en grande partie de cette politique menée par l'État britannique contre la présence d'une immigration non blanche venue du Commonwealth ${ }^{18}$.

Si la France et l'Angleterre présentent des conditions d'accès à la nationalité relativement similaires, les deux pays ont mis en œuvre des modèles d'intégration différents. Le modèle français est guidé par une conception universaliste qui vise à transformer tout étranger en citoyen français en l'espace d'une génération. Les expressions de " $\mathrm{Creu}$ setfrançais 19 " ou de " communautédes citoyens ${ }^{20}$ " ont été utilisées pour qualifier ce modèle qui prescrit le cantonnement des spécificités ethniques, religieuses et culturelles des citoyens dans la sphère privée. Si l'arrivée au pouvoir du gouvernement socialiste en mai 1981 entraîne une brève période de reconnaissance des cultures immigrées ${ }^{21}$, la fin des années 1980 est marquée par une résurgence des discours néo-assimilationnistes qui enjoignent

14. John Solomos, Race and Racism in Britain, New York, Palgrave Macmillan, 2003. 15. Patrick Weil, La République et sa diversité. Migrations, intégration, discrimination, Paris, Seuil, 2005. 16. Ce projet a été abandonné en raison de l'avis défavorable émis par le Conseil d'État. 17. Zig Layton-Henry, "Race and the Thatcher government », in Zig Layton-Henry, Paul B. Rich (dir.), Race, Government and Politics in Britain, Londres, Macmillan, 1986, pp. 73- 99. 18. Randal Hansen, Citizenship and Immigration in Post-War Britain: The Institutional Origins of a Multicultural Nation, New York, Oxford University Press, 2000. 19. Gérard Noiriel, Le creuset français : histoire de l'immigration, XIXe-XX $X^{e}$ siècle, Paris, Seuil, 1988. 20. Dominique Schnapper, La communauté des citoyens, Paris, Gallimard, 1994. 21. Narguesse Keyhani," "Les "relations interculturelles". Trajectoire sociale d'une catégorie réformatrice ", thèse de doctorat en sociologie, Cachan, École normale supérieur, 2014 ; Angéline Escafré-Dublet, Culture et immigration. De la question sociale à l'enjeu politique, 1958-2007, Rennes, Presses universitaires de Rennes, 2014. 


\section{LES CHANTIERS DE LA R}

les immigrés à cantonner les signes de leur appartenance religieuse dans l'espace privé. L'exclusion de trois lycéennes d'un collège de Creil en 1989 pour avoir refusé d'enlever leur voile avant d'entrer en cours est mise en affaire par des intellectuels qui dénoncent les signes d'appartenance religieuse et entraîne l'émergence d'un «problème de l'islam ${ }^{22}$ ». Des études ethnographiques ont exploré les conséquences pratiques de ces politiques colorblind et montré comment elle s'assimile à une politique de l'altérité pour les citoyens d'origine immigrée ${ }^{23}$.

Le modèle anglais pense au contraire l'intégration comme l'organisation étatique d'une cohabitation entre la population majoritaire et les populations immigrées. Ce modèle, qualifié de multiculturaliste ${ }^{24}$ ou de race relation $^{25}$ reconnaît les spécificités ethniques et culturelles comme des médiations dans ce processus et les autorise donc à s'exprimer dans l'espace public. Le modèle anglais est, à cet égard, proche de celui du Canada, des États-Unis et de l'Australie, et repose sur le principe d'une reconnaissance des processus de racialisation et de leurs effets sur le renforcement des inégalités. Les chercheurs ont également observé la diffusion transnationale d'un cadrage en termes de reconnaissance des droits des minorités impulsée par les organisations internationales ${ }^{26}$.

L'État britannique institutionnalise aussi, dès les années 1970, une politique de lutte contre les discriminations à travers la mise en place de la

Commission for Racial Equality (CRE). Cette agence publique dispose d'un budget de 15 millions de livres par an et d'un personnel de plus d'une centaine de travailleurs à temps plein. Elle organise des campagnes contre les discriminations, collecte et analyse les statistiques sur les discriminations, publie un rapport annuel sur le racisme en Angleterre et finance des organismes locaux pour combattre le racisme. Au-delà de son rôle d'accompagnement des victimes du racisme, elle mène

22. Thomas Deltombe, L'islam imaginaire. La construction médiatique de l'islamophobie en France, 1975-2005, Paris, La Découverte, 2005. 23. Sarah Mazouz, La République et ses autres. Politiques de l'altérité dans la France des années 2000, Lyon, ENS éd., 2017. 24. Milena Doytcheva, Le multiculturalisme, Paris, La Découverte, 2011. 25. Harry Goulbourne, Race Relations in Britain since 1945, Basingstoke, Palgrave, 1998. 26. Will Kymlicka, Multicultural odysseys: navigating the new international politics of diversity, Oxford, Oxford University Press, 2007. 
aussi des audits des industries et des départements d'État qu'elle suspecte d'agir de manière discriminatoire. Bien que le pouvoir d'agir de cette institution soit limité, son ancienneté révèle le souci précoce du gouvernement britannique pour la question des discriminations raciales.

\section{La reconnaissance de l'existence de discriminations ethno-raciales}

Les chercheurs se sont aussi intéressés aux phénomènes ségrégatifs et discriminatoires dans les politiques du logement social. En Angleterre, un pan entier de la recherche, les Race and Housing Studies, a été consacré dès les années 1960 à cette question. Des études ont identifié des phénomènes de discrimination institutionnelle dans l'attribution des logements sociaux. La Commission for Racial Equality a commandé de nombreuses études sur le logement social. En 1984, elle publie un rapport sur le quartier de Hackney à Londres, qui conclut à l'existence de stéréotypes ethniques dans l'évaluation des demandes de logements sociaux. La fin des années 1980 est marquée par la publication d'études sur la discrimination raciale dans les villes de Birmingham ${ }^{27}$ et Bedford ${ }^{28}$. Ce domaine de la recherche a néanmoins été délaissé depuis ces travaux, tant pas la recherche académique que par la $\mathrm{CRE}^{29}$.

La reconnaissance de l'existence de discriminations ethno-raciales est beaucoup plus récente en France. Des enquêtes quantitatives ont apporté des preuves que les critères ethniques affectent les parcours des immigrés et de leurs enfants dans la société française. l'Institut national de la statistique et des études économiques (INSEE) et l'Institut national d'études démographiques (Ined) ont mené conjointement en 2008 et 2009 l'enquête "Trajectoires et origines des migrants et de leurs descendants " (enquête TeO) qui établit que les personnes nées en France de parents immigrés sont plus susceptibles que les enfants de natifs d'être confrontées à des inégalités ${ }^{30}$. C'est dans le domaine de l'accès à l'emploi que les effets de l'origine ethnique semblent le plus prégnants, comme le montrent les recherches qui démontrent l'existence d'une "pénalité ethnique ancrée dans le temps » et d'une persistance des inégalités liées à l'immigration entre générations ${ }^{31}$. Les études sur les inégalités spatiales liées à l'origine signalent également une augmentation globale de la ségrégation raciale dans les grandes métropoles françaises ${ }^{32}$. Les origines affectent aussi les trajectoires des enfants d'immigrés à l'école ${ }^{33}$.

Didier Fassin a aussi retracé la reconnaissance progressive des discriminations raciales par les dispositifs institutionnels et réglementaires français ${ }^{34}$ La mise en place d'institutions comme la Haute Autorité de lutte contre les discriminations et pour l'égalité (Halde) puis le Défenseur des droits ont contribué à une progressive reconnaissance des discriminations à l'embauche et des discriminations au faciès subies par les minorités racisées dans les quartiers populaires. Mais, comme le signale Joan Stavo-Debauge, cette politique de

27. Jeff Henderson, Valerie A.Karn Race, Class and State Housing: Inequality and the Allocation of Public Housing in Britain, Aldershot, Avebury, 1987. 28. Deborah Phillips, Philip Sarre, Richard Skellington, Ethnic Minority Housing: Explanations and Policies, Aldershot, Gower, 1989. 29. Valérie Sala Pala, Discriminations ethniques: les politiques du logement social en France et au Royaume-Uni, Rennes, Presses universitaires de Rennes, 2013. 30. Chris Beauchemin, Christelle Hamel, Patrick Simon, Trajectoires et origines. Enquête sur la diversité des populations en France, Paris, Éditions de l'Ined, 2016. 31. Dominique Meurs, Ariane Pailhé, Patrick Simon, "Persistance des inégalités entre générations liées à l'immigration : l'accès à l'emploi des immigrés et de leurs descendants en France ", in Population, vol. 61, $\mathrm{n}^{\circ} 5,2006$, pp. 763-801; Roxane Silberman, Irène Fournier, "Les enfants d’immigrés sur le marché du travail. Les mécanismes d'une discrimination sélective ", in Formation emploi, $n^{\circ} 65,1999$, pp. 31-55. 32. Safi Mirna, "La dimension spatiale de l'intégration : évolution de la ségrégation des populations immigrées en France entre 1968 et 1999 ", in Revue française de sociologie, vol. 50, n 3, 2009, pp. $521-552$; Edmond Préteceille, "La ségrégation ethno-raciale a-t-elle augmenté dans la métropole parisienne ? ", in Revue française de sociologie, vol. $50, n^{\circ} 3$, 2009, pp. 489-519. 33. Mathieu Ichou, "Différences d'origine et origine des différences : les résultats scolaires des enfants d'émigrés/ immigrés en France du début de l'école primaire à la fin du collège ", in Revue française de sociologie, vol. 54, $n^{\circ} 1,2013$, pp. 5-52. 34. Didier Fassin, "L'invention française de la discrimination ", in Revue française de science politique, vol. 52, n4, $2002, \mathrm{pp} .403-423$. 
lutte contre les discriminations peine à être mise en œuvre en raison de la persistance d'un dispositif catégoriel qui continue d'individualiser le processus d'intégration en le présentant comme étant "l'affaire " exclusive des immigrés et de leurs descendants ${ }^{35}$. De même, des chercheurs ont étudié les usages ordinaires du droit de la non-discrimination et identifié des obstacles dans la possibilité pour les individus d'obtenir réparation et pour l'État d'instaurer l'égalité de traitement comme norme sociale ${ }^{36}$.

En dépit de ces transformations des modes de perception du racisme, le modèle français d'intégration et sa réaffirmation à la fin des années 1980 pèsent encore très fortement sur les réticences à mesurer - et donc à reconnaître - les inégalités ethno-raciales ${ }^{37}$. Le déficit de connaissance est flagrant si on le compare, par exemple, avec la discussion publique sur l'état des discriminations raciales outre-Atlantique ${ }^{38}$. Des chercheurs français pointent les impensés du modèle colorblind, et proposent un usage critique et analytique du concept de race ${ }^{39}$ et l'instauration d'un système de statistiques ethno-raciales pour objectiver et réduire les inégalités fondées sur l'origine ${ }^{40}$.

L'intérêt pour la discrimination raciale dans les politiques d'attribution des logements sociaux est également relativement récent en France. Les chercheurs français se sont intéressés aux usages des notions de "mixité sociale » et de "diversité » et aux pratiques discriminatoires et/ou ségrégatives qui ont été menées en leur nom ${ }^{4}$. Ces recherches ont pointé l'existence de discriminations raciales dans l'accès au logement social et mis en évidence les processus d'exclusion et de racialisation à l'œu rre dans l'accès au logement social, en rupture avec le principe de colorblindness (indifférence aux différences). Des chercheurs ont aussi réalisé des études comparatives qui montrent qu'en France comme au Royaume-Uni, la politique du logement social contribue à la construction des frontières ethniques et à la production des discriminations raciales ${ }^{42}$.

\section{Les mobilisations des immigrés et de leurs descendants : des histoires contrastées}

Une importance littérature comparative s'est intéressée aux mobilisations des immigrés et de leurs descendants. Le débat a notamment porté sur l'influence des modèles nationaux d'intégration sur les normes de mobilisations privilégiées. Plusieurs auteurs ont souligné l'émergence d'une nouvelle citoyenneté « post-nationale " qui s'étend au-delà des frontières des États-nations ${ }^{43}$. D'autres auteurs réfutent cette idée d'une transnationalisation des modes de protestation et montrent, à partir d'études comparatives, que les minorités agissent en relation avec l'environnement politique national. Ruud Koopmans et Paul Statham signalent que les revendications multiculturelles des populations musulmanes sont quasi inexistantes en

\footnotetext{
35. Joan Stavo-Debauge, "Apprêter un chemin au droit pour qu'il exerce son autorité. Confectionner des " catégories " autorisant l'action publique ", in Carnets de bord, $n^{\circ}$ 7, 2004, pp. 32-54. 36. Vincent-Arnaud Chappe, "L'égalité en procès : sociologie politique du recours au droit contre les discriminations au travail ", thèse de doctorat de sociologie, Cachan, École normale supérieure, 2013. 37. Patrick Simon, "Les statistiques, les sciences sociales françaises et les rapports sociaux ethniques et de "race" ", in Revue française de sociologie, vol. 49, $\mathrm{n}^{\circ}$ 1, août 2008, pp. 153-162. 38. Adèle Momméja, "Hyperincarcérés ", in La Vie des Idées, 13 juin 2017, http:// www.laviedesidees.fr/Hyperincarceres.html. 39. Magali Bessone, Sans distinction de race? Une analyse critique du concept de race et de ses effets pratiques, Paris, Vrin, 2013. 40. Joan Stavo-Debauge, "Apprêter un chemin au droit, op. cit. 41. Thomas Kirszbaum, "Les immigrés dans les politiques de l'habitat. Variations locales sur le thème de la diversité ", in Sociétés contemporaines, $\mathrm{n}^{\circ} 33-34,1999$, pp. 87-110 ; Sylvie Tissot, " Une " discrimination informelle " ? Usages du concept de mixité sociale dans la gestion des attributions de logements HLM ", in Actes de la recherche en sciences sociales, $n^{\circ}$ 159, 2005 ; Christine Lelévrier, "La mixité dans la rénovation urbaine : dispersion ou re-concentration? ", in Espaces et sociétés, $n^{\circ}$ 140-141, 2010, pp. 59-74. 42. Maurice Blanc, "Du logement insalubre à l'habitat social dévalorisé. Les minorités ethniques en Allemagne, France et Angleterre ", in Les annales de la recherche urbaine, $n^{\circ} 49$, 1990, pp. 37-48. 43. David Jacobson, Rights Across Borders: Immigration and the Decline of Citizenship, Baltimore, Johns Hopkins University Press, 1997 ; Saskia Sassen, «De Facto Transnationalizing of Immigration Policy », in Christian Joppke, Challenge to the Nation-State: Immigration in Western Europe and the United States, Oxford, Oxford University Press, 1998.
} 
Allemagne, alors qu'elles jouent un rôle important en Angleterre. Pour ces chercheurs, la différence entre deux pays - qui comptent une population musulmane d'importance équivalente-s'explique par la mise en place, en Angleterre, d'un système de race relation ouvrant aux minorités des opportunités pour revendiquer des droits et la reconnaissance d'une identité culturelle. L'émergence d'une Black politics parmi les communautés afrocaribéennes qui revendiquent une identité racialisée s'explique ainsi par la manière dont l'État britannique s'est construit comme un État multiculturel44. En Allemagne, au contraire, les immigrés et leurs descendants sont considérés comme étrangers à la communauté politique et ont peu de voies d'accès au politique pour revendiquer des droits sociaux et politiques 45 .

En France, les mobilisations des immigrés et de leurs descendants se sont également longtemps heurtées à un système politique réfutant toute forme de mobilisation sur des bases ethniques. La possibilité de s'organiser politiquement fut longtemps déniée aux immigrés qui ont dû attendre une loi d'octobre 1981 pour pouvoir se constituer en association ${ }^{46}$. L'arrivée de François Mitterrand au pouvoir marque une ouverture des opportunités politiques pour les mobilisations des immigrés et de leurs descendants. De nombreuses associations de jeunes d'origine immigrée sont créées à Paris et en banlieue et marquent un renouvellement générationnel de la politique immigrée. La Marche pour l'égalité et contre le racisme, initiée dans les quartiers populaires lyonnais, marque l'irruption dans l'espace public d'une jeunesse d'origine immigrée réclamant la fin des violences policières et le droit à l'égalité ${ }^{47}$. Cette période politique a pourtant été éphémère et ne s'est jamais institutionnalisée dans des politiques antidiscriminatoires. Roger Brubaker a qualifié ce moment de l'histoire politique française de « tournant différentialiste » et suggère qu'elle revêt un caractère uniquement rhétorique ${ }^{48}$. D'autres recherches empiriques ont montré que cette politique a transformé des parcours de vie et rendu possibles des trajectoires de politisation parmi la jeunesse issue de milieux populaires et immigrés. Mais ces mouvements minoritaires se sont essoufflés à la fin de la décennie 1980, en raison à la fois de dissensions internes et de l'émergence de SOS Racisme, une association crée par des leaders étudiants d'extrême gauche en quête de reconversion politique et soutenue par le parti socialiste ${ }^{49}$.

\section{Les émeutes urbaines et leur mise en signification}

Si les mouvements contestataires ont joué un rôle important dans la visibilité de la jeunesse immigrée au début des années 1980, cette période a aussi été marquée par de nombreux épisodes émeutiers. L'Angleterre connait plusieurs émeutes en 1975,1976,1981 et 1985 dans les villes de Londres, Bristol, Manchester, Birmingham, Liverpool et Leeds. Les émeutes de 1975 et 1976 peuvent être qualifiées de raciales puisqu'elles rassemblent majoritairement des jeunes hommes d'origine afro-caribéenne. La réaction immédiate du gouvernement Thatcher à ces émeutes fut de les présenter comme des actes criminels et de réfuter toute tentative de les lier aux problèmes sociaux tels que la relégation urbaine, l'inégalité raciale, le

\footnotetext{
44. Paul Statham, "Political mobilisation by minorities in Britain: Negative feedback of "race relations" ? ", in Journal of Ethnic and Migration Studies, vol. 25, $\mathrm{n}^{\circ}$ 4, 1999. 45. Ruud Koopmans, Paul Statham, "How national citizenship shapes transnationalism : A comparative analysis of migrant and minority claims-making in Germany, Great Britain and the Netherlands ", in Christian Joppke, Ewa Morawska (dir.), Toward Assimilation and Citizenship: Immigrants in Liberal Nation-States, London, Palgrave, 2003. 46. La loi du 9 octobre 1981 votée par le gouvernement de François Mitterrand abroge la nécessité de soumettre une demande d'autorisation préalable au ministère de l'Intérieur pour former une association et libéralise de facto le droit d'association pour les immigrés. 47. Abdellali Hajjat, La Marche pour l'égalité et contre le racisme, Paris, Amsterdam, 2013. 48. Rogers Brubaker, « The return of assimilation? Changing perspectives on immigration and its sequels in France, Germany, and the United States ", in Ethnic and Racial Studies, vol. 24, $n^{\circ}$ 4, 2001, pp. 531-548. 49. Philippe Juhem, «Entreprendre en politique. De l'extrême gauche au PS: la professionnalisation politique des fondateurs de SOS Racisme ", in Revue française de science politique, vol. 51, n 1, 2001, pp. 131 153.
} 
chômage et les violences policières ${ }^{50}$. Une mission d'enquête est néanmoins réunie pour comprendre les causes de l'émeute de 1982 dans le quartier londonien de Brixton. Le rapport Scarman, du nom du juge en charge de cette enquête, associe la survenue de cet épisode émeutier aux griefs des jeunes Noirs contre la police locale ${ }^{51}$. À l'instar du rapport Kerner, rédigé après les émeutes de Détroit en $1967^{52}$, ce document fait le lien entre les émeutes, l'absence de représentativité ethno-raciale de la police britannique et la relégation urbaine. Une des propositions centrales du rapport est de remédier aux conflits entre la police et les populations locales en favorisant l'embauche dans les forces de l'ordre de personnes appartenant aux minorités racisées 53

À côté de cette réponse institutionnelle, un important travail de légitimation de ces émeutes visant à leur donner une signification politique a été mené dans les cercles de militants et d'intellectuels critiques. Après les émeutes de la fin des années 1970, une campagne publique est notamment menée sous le slogan "Self-defense is no offense ». Elle a pour objectif de rendre visibles les années de discrimination raciale et de violence physique subies par les jeunes Noirs des quartiers populaires de Londres. Les intellectuels insistent de leur côté sur la condition d'aliénation de la population noire dans la société britannique et rappellent que les désordres urbains naissent d'un profond sentiment d'injustice ${ }^{54}$ John Benyon identifie ainsi cing raisons principales des désordres urbains : l'inégalité raciale et la discrimination, le taux de chômage particulièrement important parmi les hommes d'origine afro-caribéenne, la relégation urbaine, l'exclusion de la communauté politique et l'hostilité envers la police qui se rend régulièrement coupable d'abus et de harcèlement ${ }^{55}$. L'étude des conditions sociales de possibilité de l'émeute a permis de renverser l'ordre des responsabilités en pointant les inégalités sociales et raciales qui aboutissent à un phénomène "d'émeute silencieuse " (quiet riot), selon la formule suggestive d'un ouvrage paru en $1988^{56}$. Les sciences sociales britanniques ont ainsi contribué à interpréter ces émeutes comme constitutives de la condition sociale des jeunesses racisées des quartiers populaires britanniques. Ils ont ainsi contrebalancé les analyses dites du Law and order ${ }^{57}$ qui suggèrent de durcir les sanctions pénales et policières au détriment d'un traitement préventiffondé sur le développement communautaire, la médiation et la réhabilitation. La tentative de donner une signification politique aux émeutes est néanmoins spécifique à cette période de l'histoire britannique puisque les émeutes qui se sont produites à Londres en 2001 et 2017 n'ont pas fait l'objet d'un tel travail de légitimation politique de l'action émeutière ${ }^{58}$. En France, les conditions sociales qui président à la survenue des émeutes dans les quartiers populaires sont relativement similaires. Les émeutes urbaines se produisent dans les quartiers relégués lyonnais, notamment dans le quartier de la Grappinière à Vaulx-en-Velin et à la cité Olivier-deSerres à Villeurbanne. La principale réponse des pouvoirs publics consiste à mettre en place une stratégie d'intervention consistant à traiter les problèmes sociaux à partir de la production de diagnostics sur les quartiers dits "sensibles". L'État français a ainsi prétendu améliorer la situation des habitants des quartiers populaires sans

50. John Solomos, op. cit. 51. Lord Scarman, The Scarman Report: The Brixton Disorders, 10-12 April, 1981, Harmondsworth, Penguin Books, 1982. 52. Report Kerner, The 1968 Report of the National Advisory Commission on Civil Disorders, New York, Pantheon Books,

1968. 53. Andrea Rea, "Les émeutes urbaines : causes institutionnelles et absence de reconnaissance ", in Déviance et société, vol. 30 , $n^{\circ}$ 4, février 2007, pp. 463-475. 54. Stuart Hall, " Urban unrest in Britain ", in John Benyon, John Solomos (dir.), The Roots of Urban Unrest, Oxford, Pergamon Press, 1987, pp. 45-50. 55. John Benyon, "Interpretations of civil disorder ", in John Benyon, John Solomos (dir.), op. cit., pp. 23-41. 56. Fred Harris, Roger Wilkins (dir.),, Quiet Riots: Race and Poverty in the United States, New York, Pantheon Books,

1988. 57. David Dixon, Elaine Fishwick, "The law and order debate in historical perspective ", in Philip Norton (dir.), Law and order in British politics, Hampshire, Gower, 1984, pp. 21-37. 58. Gargi Bhattacharyya, Ethnicities and Values in a Changing World, Londres, Routledge, 2016. 
agir sur les inégalités sociales structurelles qui les maintiennent dans une situation de précarité socio-économique ${ }^{59}$.

Des chercheurs ont suggéré de penser ensemble les transformations de la composition des grands ensembles, le processus de relégation des populations immigrées dans les quartiers populaires et les phénomènes de violences urbaines qu'entraînent sporadiquement ces violences sociales ${ }^{60}$. Plusieurs travaux ont également tenté de penser sociologiquement les émeutes de 2005 provoquées par la mort de deux adolescents poursuivis par la police dans un transformateur à Aulnaysous-Bois ${ }^{6}$. Les violences policières et les humiliations subies par les jeunes dans les quartiers populaires apparaissent comme l'un des griefs majeurs déclenchant les épisodes émeutiers ${ }^{62}$. Des études comparatives ont également croisé l'étude du phénomène émeutier et de sa perception en France et en Angleterre63. Le milieu des années 2000 voit aussi la genèse d'une réflexion sur la recomposition des différents types d'infériorisation au croisement de la question sociale et de la question raciale ${ }^{64}$. Enfin, des recherches ethnographiques récentes sur le rapport à la religion parmi la jeunesse des quartiers populaires ont permis de complexifier la compréhension de la place de l'Islam dans les parcours de vie des populations désaffiliées ${ }^{65}$.

\section{Conclusion}

L'existence de modèles d'intégration définis nationalement et s'exerçant mécaniquement de bas en haut est remise en question par les études empiriques sur la fabrique des politiques d'immigration et les expériences concrètes des immigrés et de leurs descendants. On observe néanmoins des spécificités dans les dispositifs étatiques de lutte contre les discriminations et les formes de protestation des immigrés en France et en Angleterre qui semblent liées à l'histoire de ces deux pays. Le modèle britannique des race relations a ainsi influencé la formation d'une race politics (politique de la race) parmi les communautés afrocaribéennes. En France, l'émergence de mouvements protestataires se fait dans des contextes historiques spécifiques, comme le début des années 1980, où les enfants d'immigrés ont pu articuler leur appartenance aux classes populaires et leur origine immigrée dans des mouvements de lutte pour l'égalité. Malgré ces spécificités nationales, les deux pays connaissent aussi un mouvement convergent de fermeture de leurs frontières, de restriction des possibilités d'accès à la nationalité et d'exclusion d'une partie de leurs citoyens sur le fondement de leur origine ethnique.

59. Sylvie Tissot, L'État et les quartiers: genèse d'une catégorie de l'action publique, Paris, Seuil, 1997. 60. Stéphane Beaud, Michel Pialoux, Retour sur la condition ouvrière. Enquête aux usines Peugeot de Sochaux-Montbéliard, Paris, La Découverte, 2012 [1999] ; Violences urbaines, violence sociale, Paris, Fayard, 2013. 61. Didier Fassin, Éric Fassin, "Introduction. À l'ombre des émeutes ", in Didier Fassin, Éric Fassin (dir.), De la question sociale à la question raciale? Représenter la société française, Paris, La Découverte. 2006, pp. 5-16 ; Michel Kokoreff, "Sociologie de l'émeute", in Déviance et société, vol. 30, n 4, 2006, pp. 521-533. 62. Fabien Jobard, Jérémie Gauthier, Police. Questions sensibles, Paris, Puf, 2018. 63. Fabien Jobard, Mike King, David Waddington (dir.), Rioting in the UK and France, Portland, Willan, 2009. 64. Didier Fassin, Éric Fassin (dir.), op. cit. 65. Fabien Truong, Loyautés radicales. L'islam et les "mauvais garçons " de la nation, Paris, La Découverte, 2017 